\title{
Profound implications of COVID-19 pandemic lockdown on the Earth's ecosystem: a case study using remote sensing data
}

\author{
Nidhi Verma ${ }^{1, *}$, Nilesh Kumar ${ }^{2}$, Pooja Mishra ${ }^{1}$ and Neetesh Purohit ${ }^{1}$ \\ ${ }^{1}$ Department of Electronics and Communication Engineering, Indian Institute of Information Technology Allahabad, Prayagraj 211 015, India \\ ${ }^{2}$ Department of Biology, University of Alabama at Birmingham, 1300 University Boulevard, Birmingham, AL 35294, USA
}

\begin{abstract}
The suddenly enforced COVID-19 pandemic lockdown has impacted the Earth's environment, and several studies have reported a significant change in air pollution using remote sensing data. In this study, we highlight significant changes in the environment due to reduced human activities using MODIS, Sentinel-5 and NASA-USDA/SMAP satellite data. Critical parameters such as nitrogen dioxide, land surface temperature, surface soil moisture and normalized difference vegetation index were compared (prepandemic and post-pandemic) for six highly impacted cities around the world. This study is crucial for environmental management to maintain sustainability of the Earth's ecosystem.
\end{abstract}

Keywords: Case study, COVID-19 pandemic, environmental parameters, lockdown impact, remote sensing data.

TIMELY mapping of land surface temperature (LST), surface soil moisture (SSM), nitrogen dioxide $\left(\mathrm{NO}_{2}\right)$ concentration and normalized difference vegetation index (NDVI, a vegetation greenness indicator) is important to understand the Earth's ecosystem. Satellite data are available since 1980 onwards in many cases; therefore, they provide a tool for carrying out historical analyses and monitoring the changes that have occurred in the atmosphere. In the present study, temperature, soil moisture, $\mathrm{NO}_{2}$ and vegetation parameters, which are crucial for understanding the Earth's ecosystem and its sustainability, have been analysed for the pre- and post-COVID-19 pandemic lockdown phase. The impact of anthropogenic activities on the environment was the worst ever before the imposition of the lockdown in 2020 in various parts of the world. Wuhan, one of the most populous cities in central China reported its first confirmed novel coronavirus infection case in the last week of December 2019 and became the first ever COVID-19 epicenter ${ }^{1}$. On 11 March 2020, the World Health Organization (WHO) classified the COVID-19 epidemic as a global pandemic ${ }^{2}$. Within six months of its incidence, the disease had

\footnotetext{
*For correspondence. (e-mail: nidhiverma.iiita@gmail.com)
}

spread to more than 200 countries, or especially highly polluted urban areas/cities ${ }^{3-8}$. Within six months, more than 7 million people were infected, and over 400,000 people lost their lives ${ }^{9}$. As the first country affected by COVID-19, China imposed its first lockdown on 23 January 2020. Eventually, other countries around the globe started to take the necessary steps to check the infection. The preventive measures included as social distancing, washing hands with a sanitizer and stay-at-home orders, closing nonessential businesses and regional lockdown leading to an abrupt decrease in anthropogenic activities $^{10-13}$.

The reduction in anthropogenic activities had a significant impact on air pollution around the globe during the lockdown period. Various studies reported significant decline in $\mathrm{NO}_{2}$ in China ${ }^{14-16}$. $\mathrm{NO}_{2}$ is a smelly gas and an air pollutant; it also impacts animal health ${ }^{4,17}$. The decreased concentration level of $\mathrm{NO}_{2}$ in the atmosphere is an indicator of good air quality ${ }^{18}$. Nitrogen $(\mathrm{N})$ is an essential component of air and one of the driving factors of the Earth's ecosystem ${ }^{19}$. Abiotic factors such as LST, SSM and vegetation play a pivotal role in the biogeochemical cycle in the Earth's ecosystem ${ }^{20-23}$.

Studies related to the impact of COVID-19 pandemic lockdown on the environment are mainly based on airpollution analysis. Hence, in the present study, we aim to explore significant changes observed in the environment, i.e. $\mathrm{NO}_{2}$ and abiotic (LST, SSM, NDVI) parameters due to the COVID-19 lockdown and related reduced human activities. For this, multi-date and multi-satellite, remotesensing data pertaining to $\mathrm{NO}_{2}$, LST, NDVI and SSM from six major cities of the world, viz. Bergamo, Qom, New York, New Delhi, Madrid and Wuhan were collected. For a balanced juxtaposition, we analysed datasets collected before and after or during the lockdown period for resembling time points.

\section{Materials and methods}

Study area

We had collected data from six different countries that were greatly impacted by COVID-19 and had restricted 


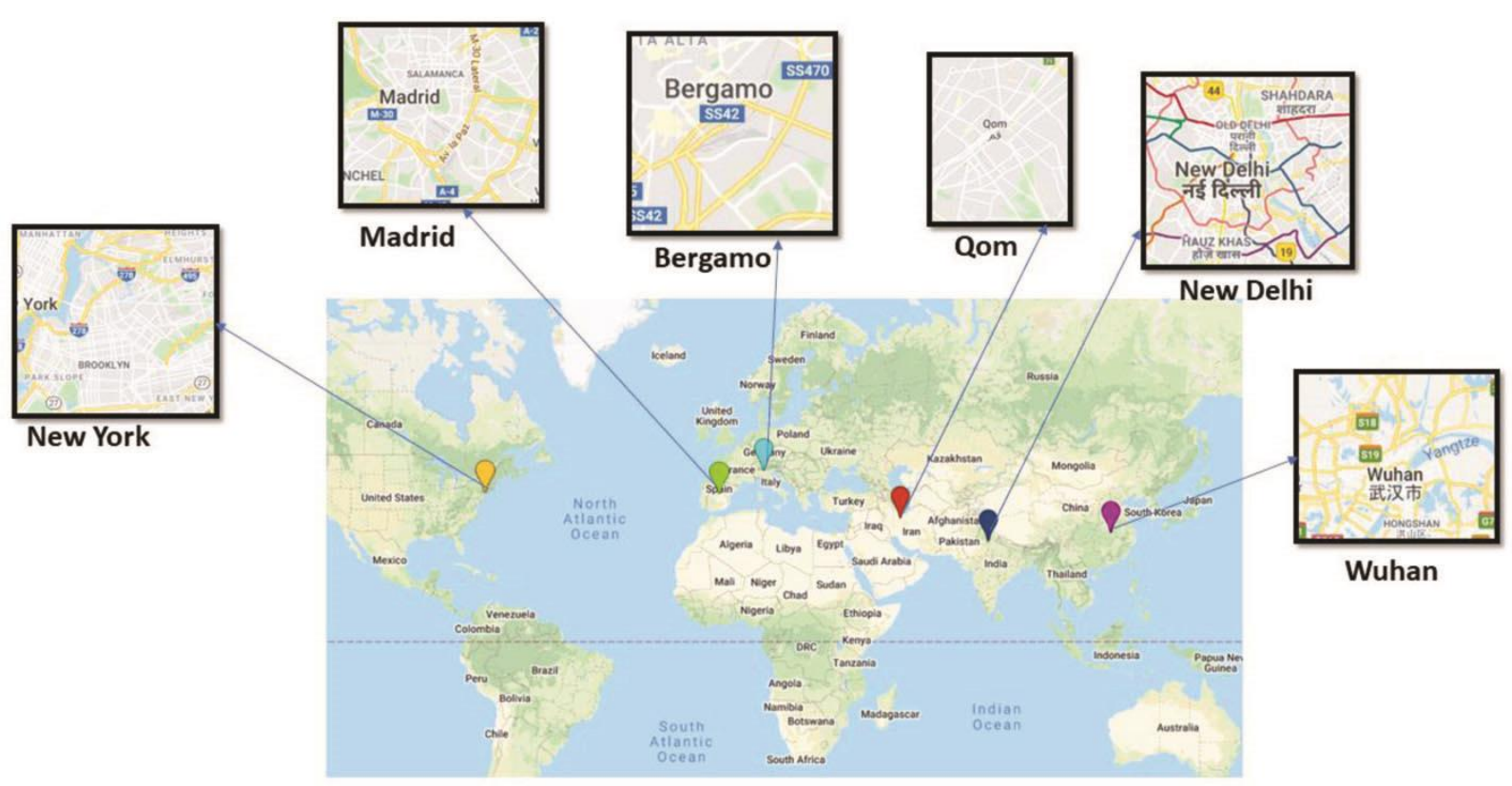

Figure 1. Major COVID-19 affected epicentres around the globe.

some forms of human activity. Wuhan is the first epicentre of the pandemic and was the first city where lockdown was imposed by China. New York, USA, registered the highest number of COVID-19 cases by the end of June 2020. New Delhi, India, the second largest city in the world with a population of more than 29 million, reported 44,000 confirmed COVID-19 cases by the third week of June 2020. Strict lockdown was imposed in New Delhi by the Government of India starting from 25 March 2020 to 31 May 2020. Bergamo city in northern Italy was one of the worst hit by COVID-19. About 1 million people were reported COVID-19 cases by the second week of May 2020. On 21 February 2020, lockdown was imposed in Bergamo. Madrid, the capital of Spain, reported more than 70,000 COVID-19 cases, and was the worst hit city in that country. Iran became the second epicentre of COVID-19 after China. Qom city in Iran registered $\sim 7178$ cases of COVID-19 by the middle of June 2020. A detailed description of the study area, including the duration for which data was collected, is given in the Supplementary Table 1. Figure 1 shows the locations of the selected study area in the world, and related shape files are given in the Supplementary Table 2.

\section{Data collection method}

Data for the study were procured from several satellite data repositories. The details are as follows: LST data (MODIS 11A1) were collected using Google Earth Engine catalogue ${ }^{24}$. The MODIS 11A1 Version 6 product provides daily per pixel land surface temperature and emissivity (LST\&E) with $1 \mathrm{~km}$ spatial resolution in a
1200 by $1200 \mathrm{~km} \operatorname{grid}^{25}$. The pixel temperature value is derived from the 'MODIS 11_L2' swath product.

The NDVI data were collected and its processing was done using MODIS/006/MCD43A4 (ref. 25). The NDVI is generated from the near infrared (NIR) and red bands of each scene. The mathematical equation for NDVI is given as below

$$
\mathrm{NDVI}=\frac{(\mathrm{NIR}-\mathrm{Red})}{(\mathrm{NIR}+\mathrm{Red})}
$$

The NDVI value varies from -1.0 to 1.0 . A value close to 1.0 represents a high vegetation index and a value close to -1.0 represents a water region. The NDVI value 0 represents bare land area.

The Sentinel-5 $\mathrm{NO}_{2}$ column density data were used to obtain $\mathrm{NO}_{2}$ concentration in the environment. The data were collected using the Google Earth Engine, and the data provider was the European Space Agency. The $\mathrm{NO}_{2}$ columns were derived using TROPOMI's UV (ultraviolet)VIS (visible) spectrometer backscattered solar radiation measurements in the 405-465 nm wavelength range ${ }^{26}$.

The NASA-USDA global soil moisture and the NASA-USDA SMAP global soil moisture datasets are available in the Google Earth Engine catalogue. The spatial resolution of these datasets is $0.25^{\circ} \times 0.25^{\circ}$. The datasets include surface and subsurface soil moisture $(\mathrm{mm})$, soil moisture profile (\%), and surface and subsurface soil moisture anomalies. Values around 0 indicate typical moisture conditions, while positive and negative values indicate extreme wet surface (soil moisture conditions are above average) and dry surface (soil moisture conditions are below average) respectively ${ }^{27}$. 

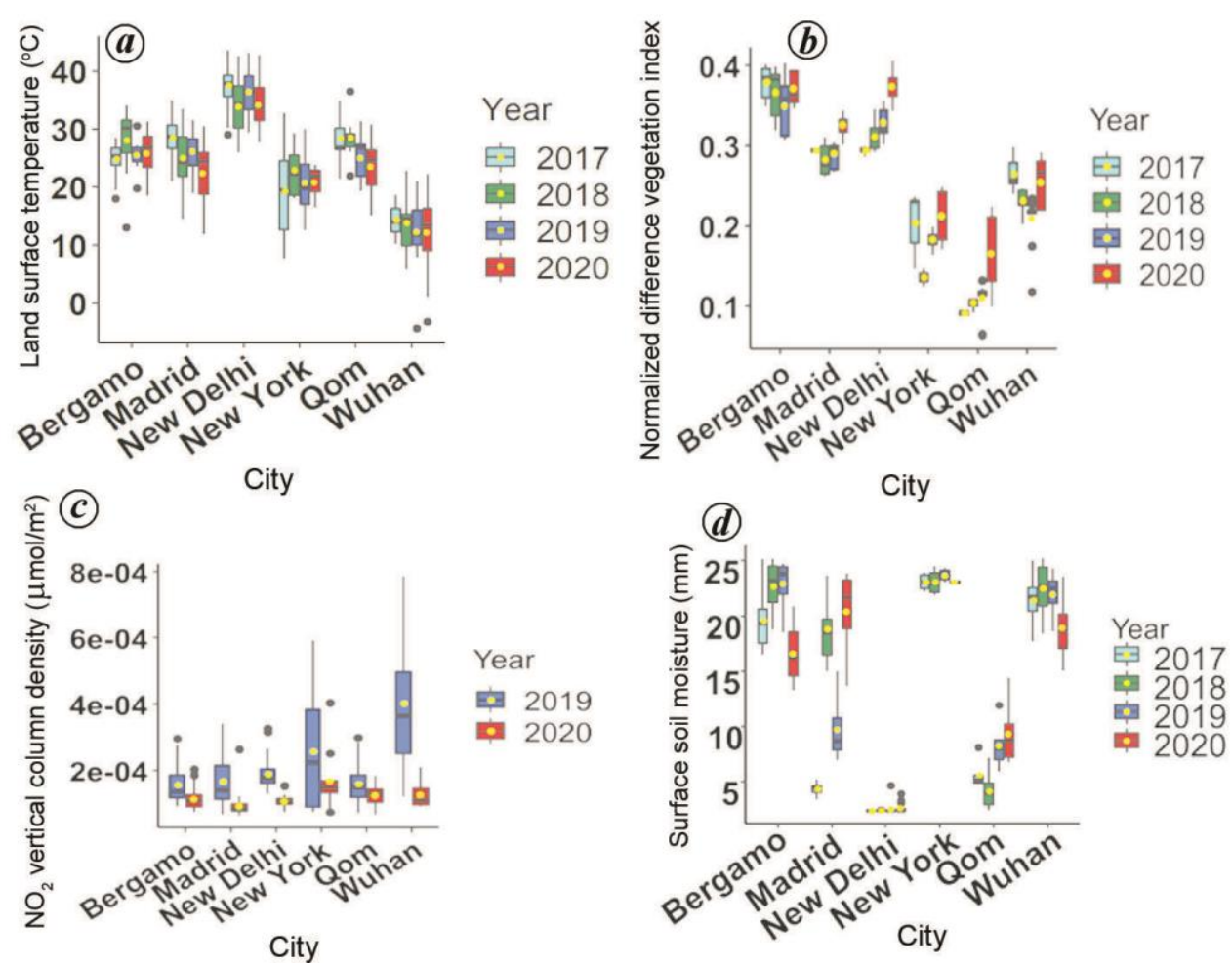

Figure 2. (a) Land surface temperature (LST) $\left({ }^{\circ} \mathrm{C}\right),(\boldsymbol{b})$ normalized difference vegetation index (NDVI), (c) nitrogen dioxide $\left(\mathrm{NO}_{2}\right)\left(\mu \mathrm{mol} / \mathrm{m}^{2}\right),(\boldsymbol{d})$ surface soil moisture $(\mathrm{SSM} ; \mathrm{mm})$ from six major cities of the world during the lockdown period or least human activity months (2020) and pre-pandemic years (2017, 2018, 2019). Different years are indicated in different colours for each city grouped together. Mean is ind icated by yellow colour.

\section{Results and discussion}

Results from pattern change in $\mathrm{LST}, \mathrm{NDVI}, \mathrm{NO}_{2}$, SSM during pre- and post-lockdown, ANOVA and Tukey's test analysis

The level of LST, NDVI, $\mathrm{NO}_{2}$ and SSM was analysed for the six different cities. LST, NDVI and SSM data were collected from 2017 to $2020 . \mathrm{NO}_{2}$ data were collected only for two years, i.e. for 2019 and 2020 (Sentinel-5 started coverage from 30 April 2018). For each year, data were collected between February and May since these are the months during which lockdown was the most effective or human activities were the least. Figure $2 a$ shows the variation of LST for the six cities grouped as a candle plot. As the viral infection began to spread rapidly during the first week of February 2020, social distancing was imposed or recommended by the competent authorities. The implementation of social distancing and lockdown were enforced everywhere. On an average, LST for 2020 was found to be less compared to the previous three years for all cities except Bergamo. When compared to 2019 and 2017, the mean LST value for 2020 was similar, which might be because of an outlier in the data. One of the reasons to explain this disparity could be the failure to implement a strict lockdown in Bergamo.
ANOVA test was also performed to estimate significant interactions between the chosen year and the city. The $P$-value for the year, city and year: city was $7.28 \mathrm{e}$ $06,<2 \mathrm{e}-16$ and 0.00786 respectively.

The $P$-value was less and significant for yearwise interactions. To compare different years altogether the Tukey's honest significance test was performed (Supplementary Table 3 ). The adj. $P$-value for the pair of years 2020-2017, 2020-2018 and 2020-2019 was 0.0000145, 0.0049087 and 0.0001648 respectively. These statistical results suggest that LST for 2020 is significantly different compared to the previous years.

NDVI is an index to measure chlorophyll content in healthy plants. The mean NDVI index was found to be increased for 2020 compared to the three previous years for Wuhan, Madrid, Qom and New Delhi.

Whereas in the case of New York, NDVI was higher compared to only the last two years. In the case of Bergamo, NDVI was highest for 2017, and it gradually decreased and again started to recover in 2020 (Figure $2 b$ ). Tukey's yearwise comparison (summarized in the Supplementary Table 4), also supports this observation the adj. $P$-value was the least $(\sim 0)$ in 2020 compared to the three previous years.

The $\mathrm{NO}_{2}$ level was significantly low compared to 2019 (Figure $2 c$ ) and the $P$-value was found to be $\sim 0$ for Tukey's 

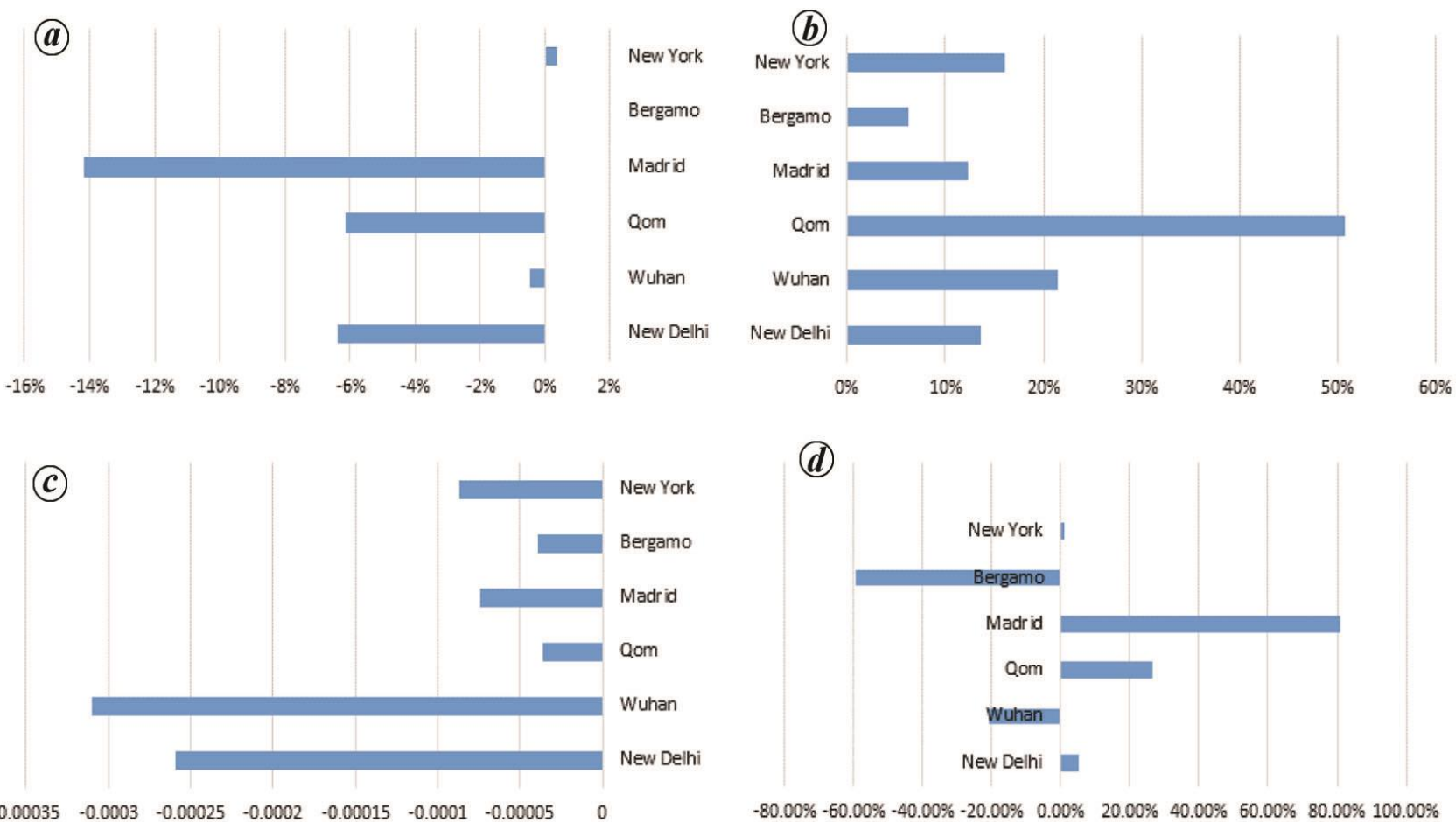

Figure 3. The percentage change in different environmental parameters: $(\boldsymbol{a})$ change (\%) in LST, $(\boldsymbol{b})$ change (\%) in NDVI, $(\boldsymbol{c})$ change $(\%)$ in $\mathrm{NO}_{2},(\boldsymbol{d})$ change (\%) in SSM for 2020-19 during pre-lockdown and post-lockdown phases in the New York, Bergamo, Madrid, Qom, Wuhan and New Delhi.

Table 1. Results on the basics of change in land surface temperature (LST), normalized difference vegetation index (NDVI), nitrogen dioxide $\left(\mathrm{NO}_{2}\right)$ and surface soil moisture (SSM)

\begin{tabular}{lcccccc}
\hline & \multicolumn{5}{c}{ Major epicentre } \\
\cline { 2 - 6 } Parameters & Bergamo & Madrid & New Delhi & New York & Qom & Wuhan \\
\hline LST & NI & IM & IM & IM & IM & IM \\
NDVI & IM & IM & IM & IM & IM & IM \\
NO2 & IM & IM & IM & IM & IM & IM \\
SSM & NI & IM & IM & NI & IM & NI \\
Remarks on the environmental conditions during & Improved & Improved & Improved & Improved & Improved Improved \\
the lockdown period based on selected & & & & & & \\
$\quad$ & & & & & & \\
\hline
\end{tabular}

NI, No improvement; IM, Improvement.

(Supplementary Table 5). The significantly decreased levels of $\mathrm{NO}_{2}$ suggest several fold decrease in air pollution.

We observed that SSM level had significantly improved in 2020 in all the cities under study as compared to 2019 and 2017 (Figure 2d) except Bergamo and Wuhan. The yearwise comparisons of the results (Supplementary Table 6), indicate that 2020 shows a significant difference from 2017. For the last three years, viz. $2017,2018,2019$, the combination pair of 2020 2017 showed the significant low value of adj.P.

Overall the statistical analysis (Tukey's test) suggests that the lockdown has brought a significant change in all four parameters for selected city areas as compared to the previous three years.
Results from percentage change in LST, NDVI, $\mathrm{NO}_{2}$, and SSM for pre-and post-pandemic time interval (2020 and 2019)

The results from Tukey's analysis clearly indicate that 2020 post-pandemic phase is significantly different from the 2019 pre-pandemic phase. Figure $3 a$ shows the percentage change in LST for 2020-2019 (for the time interval as given in Supplementary Table 1). From this, negative changes in LST percentage for all cities are observed except Bergamo. The changes are not favourable for Bergamo, because they may have not followed strict lockdown rules, and some human activities may have continued during the lockdown period. As shown in 
Figure $3 a$, on an average, LST in 2020 was less during the post-pandemic phase. However, there is a positive change in LST New York. This indicates that some human or industrial activities continued during the lockdown phase. Madrid, Qom, Wuhan and New Delhi show a significant decline in the LST percentage in 2020 compared to 2019 (Figure $3 a$ ).

Figure $3 b$ shows the percentage change in NDVI for 2020-2019 (for the time interval as given in Supplementary Table 1). The positive changes in NDVI can be observed, which implies that vegetation health has improved after imposing the lockdown for the selected cities.

Figure $3 c$ shows negative change in percentage of $\mathrm{NO}_{2}$ for 2020-2019 (for the time interval as given in Supplementary Table 1). From this, it has been observed that due to reduced human activities and industrial pollution during the lockdown, the concentration of $\mathrm{NO}_{2}$ has reduced globally. Significant negative changes are seen in Wuhan (72\%), New Delhi (72\%), Madrid (45\%) and New York $(35 \%)$.

The results from SSM were observed for the pre(2019) and post-pandemic (2020) phase. For a better ecosystem, SSM should be high for good vegetation health. Figure $3 d$ shows the positive change in percentage of SSM for 2020-2019 (for the time interval, as mentioned in Supplementary Table 1). The positive changes can be seen in all cities, except Bergamo, Wuhan and very less change in New York for the considered shapefile.

Table 1 summarizes results of analysis of LST, NDVI, $\mathrm{NO}_{2}$ and SSM. In Table 1, remarks have been given based on the results shown in Figure $3 a-d$. The overall results support that the environmental conditions were improved in Madrid, New Delhi and Qom. However, the results of all parameters for Wuhan show a positive impact, except SSM. The results from Bergamo and New York show that the environment started healing itself due to reduction in human and industrial activities. Overall, the lockdown shows a positive impact on the Earth's environment for the selected shapefile as given in Supplementary Table 2. The additional spatial map results of LST, NDVI, $\mathrm{NO}_{2}$ and SSM for large areas of all selected cites are given in the Supplementary Figures 1-4 respectively. These maps also show significant changes for the pre- and post-pandemic phase.

\section{Conclusion}

In conclusion, we have performed a comprehensive study on the effect of COVID-19 lockdown in six major epicentres of the world: New Delhi, Wuhan, Qom, Madrid, Bergamo and New York using remote-sensing data by analysing $\mathrm{NO}_{2}$ concentration and abiotic parameters, viz. LST, NDVI and SSM. A significant reduction in the concentration of $\mathrm{NO}_{2}$ was observed for all COVID-19 epi- centres. This is due to the controlled emission of major air pollutants, which can significantly minimize several health issues such as respiratory-related problems, cardiovascular illness, asthma, premature death, etc. The vegetation greenness parameter NDVI was improved for all cities during the lockdown phase. The average LST was reduced overall, except Bergamo during the lockdown. SSM was increased for all COVID-19 epicentres, except Wuhan. The increase in NDVI, SSM and reduction in LST can significantly improve vegetation health. The overall findings indicate that the COVID-19 pandemic is indeed an unfortunate event with a positive outcome in favour of the Earth's environment. On the one hand, it is a global pandemic and a serious threat to human health; it has halted economic activities. While on the other hand, it helped nature to replenish itself from anthropogenic climate change due to improvement in the environmental parameters. We have observed this positive impact on the Earth's environment, which may be useful to concerned institutions and authorities to manage our precious natural resources, and human and industrialrelated activities in the future to improve environmental and human health conditions.

Disclosure statement: The authors declare no competing interests and also no financial interests.

1. Gorbalenya, A. E. et al., Coronaviridae Study Group of the International Committee on Taxonomy of Viruses, The species severe acute respiratory syndrome-related coronavirus: classifying 2019$\mathrm{nCoV}$ and naming it SARS-CoV-2. Nat. Microbiol., 2020, 5(4), 536-544.

2. Sohrabi, C. et al., World Health Organization declares global emergency: a review of the 2019 novel coronavirus (COVID-19). Int. J. Surg., 2020, 76, 71-76.

3. Domingo, J. L., Marquès, M. and Rovira, J., Influence of airborne transmission of SARS-CoV-2 on COVID-19 pandemic. A review. Environ. Res., 2020, 188, 109861.

4. Bherwani, H. et al., Valuation of air pollution externalities: comparative assessment of economic damage and emission reduction under COVID-19 lockdown. Air Qual., Atmos. Health, 2020, 13(6), 683-694.

5. Gautam, S. and Hens, L., SARS-CoV-2 pandemic in India: What might we expect?, Environ., Develop. Sustain., 2020, 22, 3867-3869.

6. Sarkodie, S. A. and Owusu, P. A., Global assessment of environment, health and economic impact of the novel coronavirus (COVID-19). Environ., Develop. Sustain., 2021, 23, 5005-5015.

7. Aydın, S. et al., Environmental impact of coronavirus (COVID19) from Turkish perceptive. Environ., Develop. Sustain., 2021, 23(5), 7573-7580.

8. Owusu, P. A. and Sarkodie, S. A., Global estimation of mortality, disability-adjusted life years and welfare cost from exposure to ambient air pollution. Sci. Total Environ., 2020, 742, 140636.

9. WHO, Coronavirus disease (COVID-19) pandemic. 2020; https:// www.who.int/emergencies/diseases/novel-coronavirus-2019 (accessed on 15 July 2020).

10. Li, L. et al., Air quality changes during the COVID-19 lockdown over the Yangtze River Delta Region: an insight into the impact of human activity pattern changes on air pollution variation. Sci. Total Environ., 2020, 732, 139282. 


\section{RESEARCH ARTICLES}

11. Mandal, I. and Pal, S., COVID-19 pandemic persuaded lockdown effects on environment over stone quarrying and crushing areas. Sci. Total Environ., 2020, 732, 139281.

12. Siciliano, B. et al., Increased ozone levels during the COVID-19 lockdown: analysis for the city of Rio de Janeiro, Brazil. Sci. Total Environ., 2020, 737, 139765.

13. Eroğlu, H., Effects of COVID-19 outbreak on environment and renewable energy sector. Environ., Develop. Sustain., 2020, 23, 4782-4790.

14. NASA, Airborne nitrogen dioxide plummets over China, 2 March 2020; https://earthobservatory.nasa.gov/images/146362/airbornenitrogen-dioxide-plummets-over-china; Available from: https:// earthobservatory.nasa.gov/images/146362/airborne-nitrogendioxide-plummets-over-china (accessed on 15 July 2020).

15. Muhammad, S., Long, X. and Salman, M., COVID-19 pandemic and environmental pollution: a blessing in disguise? Sci. Total Environ., 2020, 728, 138820.

16. Lal, P. et al., The dark cloud with a silver lining: assessing the impact of the SARS COVID-19 pandemic on the global environment. Sci. Total Environ., 2020, 732, 139297.

17. Yang, Z. et al., Acute effects of air pollution on the incidence of hand, foot, and mouth disease in Wuhan, China. Atmos. Environ., 2020, 225, 117358

18. Moshammer, H. et al., Nitrogen-dioxide remains a valid air quality indicator. Int. J. Environ. Res. Publ. Health, 2020, 17(10) 3733.

19. Vitousek, P. M. and Howarth, R. W., Nitrogen limitation on land and in the sea: how can it occur? Biogeochemistry, 1991, 13(2), $87-115$.

20. Lin, X. et al., Discriminating surface soil inorganic nitrogen cycling under various land uses in a watershed with simulations of energy balanced temperature and slope introduced moisture. J. Hydrol., 2020, 587, 124950.
21. Yu, S. and Ehrenfeld, J. G., Relationships among plants, soils and microbial communities along a hydrological gradient in the New Jersey Pinelands, USA. Ann. Bot., 2010, 105(1), 185-196.

22. Ahmed, I. U. et al., Soil moisture integrates the influence of landuse and season on soil microbial community composition in the Ethiopian highlands. Appl. Soil Ecol., 2019, 135, 85-90.

23. Schlesinger, W. H., On the fate of anthropogenic nitrogen. Proc. Natl. Acad. Sci., 2009, 106(1), 203-208.

24. Gorelick, N., Google Earth Engine. In EGU General Assembly Conference Abstracts, 2013.

25. Daac, L., Land Cover Type Yearly L3 Global 500 m SIN Grid (MCD12Q1). Land Processes Distributed Active Archive Center (LP DAAC), located at the US Geological Survey (USGS) Earth Resources Observation and Science (EROS) Center (lpdaac. usgs.gov), Sioux Falls, 2009; https://lpdaac.usgs.gov/lpdaac/ products/modis_products_table/land_cover/yearly_13_global_500_ $\mathrm{m} / \mathrm{mcd} 12 \mathrm{q} 1$ (accessed on 20 June 2020).

26. Griffin, D. et al., High-resolution mapping of nitrogen dioxide with TROPOMI: First results and validation over the Canadian oil sands. Geophys. Res. Lett., 2019, 46(2), 1049-1060.

27. Entekhabi, D. et al., The soil moisture active passive (SMAP) mission. Proc. IEEE, 2010, 98(5), 704-716.

ACKNOWLEDGEMENT. We thank the Ministry of Human Resource Development, Government of India for providing a scholarship for the first author, and Google Earth Engine for providing free remote sensing data for carrying out this work.

Received 10 November 2020; revised accepted 5 April 2021

doi: $10.18520 / \mathrm{cs} / \mathrm{v} 121 / \mathrm{i} 1 / 115-120$ 